\title{
Das Marie-Bamberger-Syndrom als Fingerzeig auf ein NSCLC: vier Fälle im Lichte der aktuellen Literatur
}

\author{
Hypertrophic Pulmonary Osteoarthropathy as a Cue for NSCLC: \\ Four Cases in the Light of the Current Literature
}

Autoren

Institut
M. Lommatzsch, P. Julius, W. Lück, A. Bier, J. C. Virchow

Abteilung für Pneumologie, Universität Rostock eingereicht 13.10.2011

akzeptiert 18. 10. 2011

\section{Bibliografie \\ Dol http://dx.doi.org/ \\ 10.1055/s-0031-1291476 \\ Online-Publikation: 16.1.2012 \\ Pneumologie 2012; 66: 67-73 \\ (c) Georg Thieme Verlag KG \\ Stuttgart · New York \\ ISSN 0934-8387}

\section{Korrespondenzadresse}

PD Dr. Marek Lommatzsch

Abteilung für Pneumologie Zentrum für Innere Medizin, Universität Rostock

Ernst-Heydemann-Str. 6

18057 Rostock

marek.lommatzsch@med.unirostock.de

\section{Zusammenfassung \\ $\nabla$}

Das Marie-Bamberger-Syndrom (Synonym: hypertrophe pulmonale Osteoarthropathie) tritt mit einer Inzidenz von 1-5\% bei Patienten mit nichtkleinzelligem Bronchialkarzinom (NSCLC) auf. In seiner vollständigen Form treten Trommelschlegelfinger (meist ohne Vorliegen einer Zyanose) und Knochen- und Gelenkschmerzen auf. Anhand der hier dargestellten Fälle wird verdeutlicht, dass dieses paraneoplastische Syndrom zeitlich vor typischen Tumorsymptomen auftritt, jedoch auch heute noch selten erkannt wird. Wird die Symptomkonstellation Trommelschlegelfinger und Knochenschmerz frühzeitig erkannt, lässt sich ein NSCLC in einem früheren Tumorstadium diagnostizieren - daher ist es von großer klinisch-praktischer Bedeutung. Neben der Falldarstellung gibt der Artikel einen Überblick über die aktuellen Vorstellungen zur Pathogenese und Therapie dieses Syndroms.

\section{Einleitung \\ $\nabla$}

Das Pierre-Marie-Bamberger-Syndrom oder Marie-Bamberger-Syndrom (Synonym im englischen Schrifttum: hypertrophic pulmonary osteoarthropathy) kann bei Patienten mit nichtkleinzelligen Bronchialkarzinomen (Non-small cell lung cancer, NSCLC) als paraneoplastisches Syndrom auftreten $[1,2]$. Dieses Syndrom ist von großer diagnostischer Bedeutung, da es eine frühere NSCLC-Erstdiagnose ermöglicht und zudem Patienten unnötige orthopädische Untersuchungen und Behandlungen ersparen kann. Zum Marie-BambergerSyndrom gab es bereits einen Fall-Bericht in der Pneumologie im Jahr 1990 [3]. Seitdem sind eine Reihe weiterer Publikationen zu diesem Syndrom erschienen. In dem vorliegenden Artikel sollen 4 aktuelle Fälle eines Marie-Bamberger-Syndroms bei NSCLC beschrieben und vor dem Hintergrund der gegenwärtigen Literatur beleuchtet werden.

\section{Abstract \\ $\nabla$}

Hypertrophic pulmonary osteoarthropathy (often referred to as Marie-Bamberger syndrome) occurs in $1-5 \%$ of all patients with non-small cell lung cancer (NSCLC) as a paraneoplastic syndrome. The complete syndrome is characterised by clubbing of the fingers and toes (often without hypoxia) and pain in the joints and tubular bones. On the basis of four clinical cases, this article shows that this syndrome can precede tumourspecific symptoms and that it is still often overlooked by physicians. An early suspicion of this syndrome is of great clinical value because it can lead to a diagnosis of NSCLC at an earlier tumour stage. In addition to the case reports, the current literature on hypertrophic pulmonary osteoarthropathy is reviewed in this article, with special reference to pathogenetic concepts und to therapeutic options.

\section{Fall 1}

$\nabla$

Es handelt sich um einen 58-jährigen Mann, der hauptamtlicher Betriebsrat einer Rostocker Werft war (ursprünglicher Beruf: Dreher). Er hatte bis zu seinem 54. Lebensjahr 60 PY geraucht. Vorerkrankungen oder eine Dauermedikation wurden verneint. Seit 8 Monaten vor Diagnosestellung traten Knochen- und Gelenkbeschwerden, insbesondere jedoch quälende Kniebeschwerden beidseits auf. Kurz darauf kam es zu einer Ausbildung von Trommelschlegelfingern ( $\bullet$ Abb. 1 u. $\odot$ Abb. 2). Bezüglich der Knieschmerzen erfolgten mehrere Arthroskopien und arthroskopische Behandlungen, ohne dass eine Diagnose gefunden wurde oder eine Besserung eintrat. Therapieversuche mit diversen NSAR hatten nur einen mäßigen Erfolg. Zwei Monate vor Diagnosestellung gewann der Patient einen „Indoor-Cycling“-Wettbewerb und berichtete, dass auch in dieser Belastungs- 
situation keine besonderen respiratorischen Beschwerden auftraten (nur die Gelenkbeschwerden machten ihm zu schaffen). Eine B-Symptomatik wurde verneint. In der vom behandelnden Orthopäden zur weiteren Abklärung anberaumten Skelettszintigrafie zeigte sich ein typisches Anreichungsmuster wie bei einer hypertrophen Osteoarthropathie ( $\bullet$ Abb.3). In der konventionellen Bildgebung der Röhrenknochen wurde eine typische Verdickung des Periosts nachgewiesen ( $\bullet$ Abb.3). Daraufhin wurde eine Bildgebung des Thorax empfohlen, im Röntgen-Thorax und im CTThorax wurde eine große Raumforderung (mit Kavernenbildung) im rechten Oberlappen entdeckt $(\bullet$ Abb.4).

Es wurde ein Adenokarzinom der Lunge (G2) diagnostiziert, eine Abklärung bezüglich des EGFR-Status erfolgte zum damaligen Zeitpunkt (im Jahr 2008) nicht. Das Staging erbrachte die Tumorformel T4N2Mx, da eine fragliche Metastase in der 9. Rippe rechts gefunden wurde (ansonsten kein Nachweis von Fernmetastasen). Nach simultaner Radiochemotherapie folgte eine ambulante Chemotherapie mit Carboplatin und Navelbine. Darunter kam es

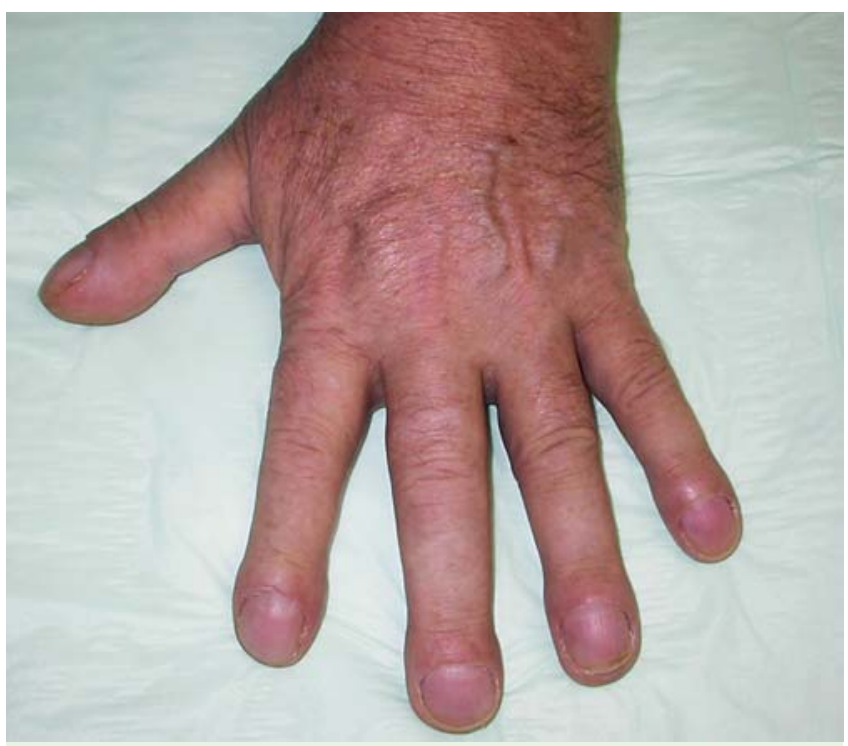

Abb. 1 Hand des Patienten im Fall Nr. 1. zu einem Tumor-Regress mit Ausbildung einer großen Kaverne im rechten Oberlappen ( $\bullet$ Abb.4). Acht Monate nach Therapiebeginn kam es zu Brustwirbelkörperfrakturen, der Patient wurde mit einem Fixateur interne neurochirurgisch versorgt. Unter dem Verdacht auf pathologische Fraktur im Rahmen einer neuaufgetretenen Metastasierung wurde die Therapie auf eine Monotherapie mit Pemetrexed umgestellt. Im Re-Staging nach 6 Monaten Pemetrexed-Therapie fand sich kein sicherer Hinweis auf Lokalrezidiv oder Fernmetastasierung. Auf Wunsch des Patienten wurde eine Therapiepause eingelegt. Im weiteren Verlauf kam es zu einer therapierefraktären Aspergillus-Infektion in der Tumorkaverne. Im PET-CT fand sich Rest-Tumor-Aktivität nur am Boden der Kaverne. Eine Lungenteilresektion war aufgrund der geringen Diffusionskapazität und des schlechten Allgemeinzustandes nicht möglich. Der Patient verstarb 20 Monate nach Erstdiagnose im Rahmen der pulmonalen Infektion. Während der gesamten Tumor-Therapie kam es zu keiner Rückbildung der Trommelschlegelfinger.

\section{Fall 2}

$\nabla$

Es handelt sich um eine 44-jährige Frau, die zunächst als Kellnerin gearbeitet hatte und seit 10 Jahren als Furnierholz-Trocknerin tätig war. Sie hatte bis zuletzt insgesamt 30 PY geraucht. Vorerkrankungen oder eine Dauermedikation wurden verneint. Seit 10 Monaten vor Diagnosestellung bemerkte die Patientin eine allgemeine Schwäche und einen ungewollten Gewichtsverlust.

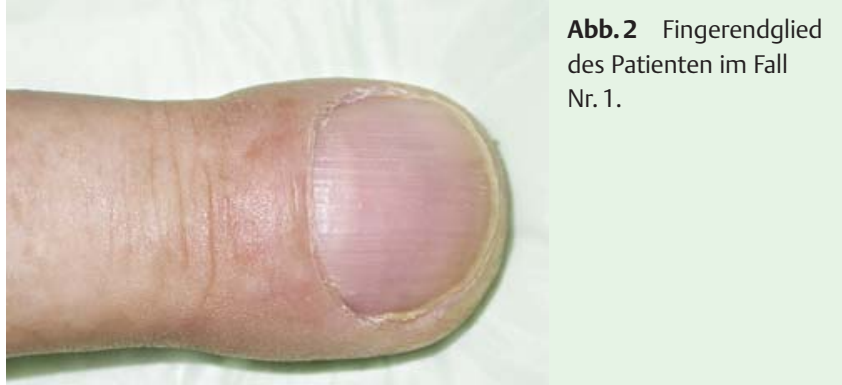

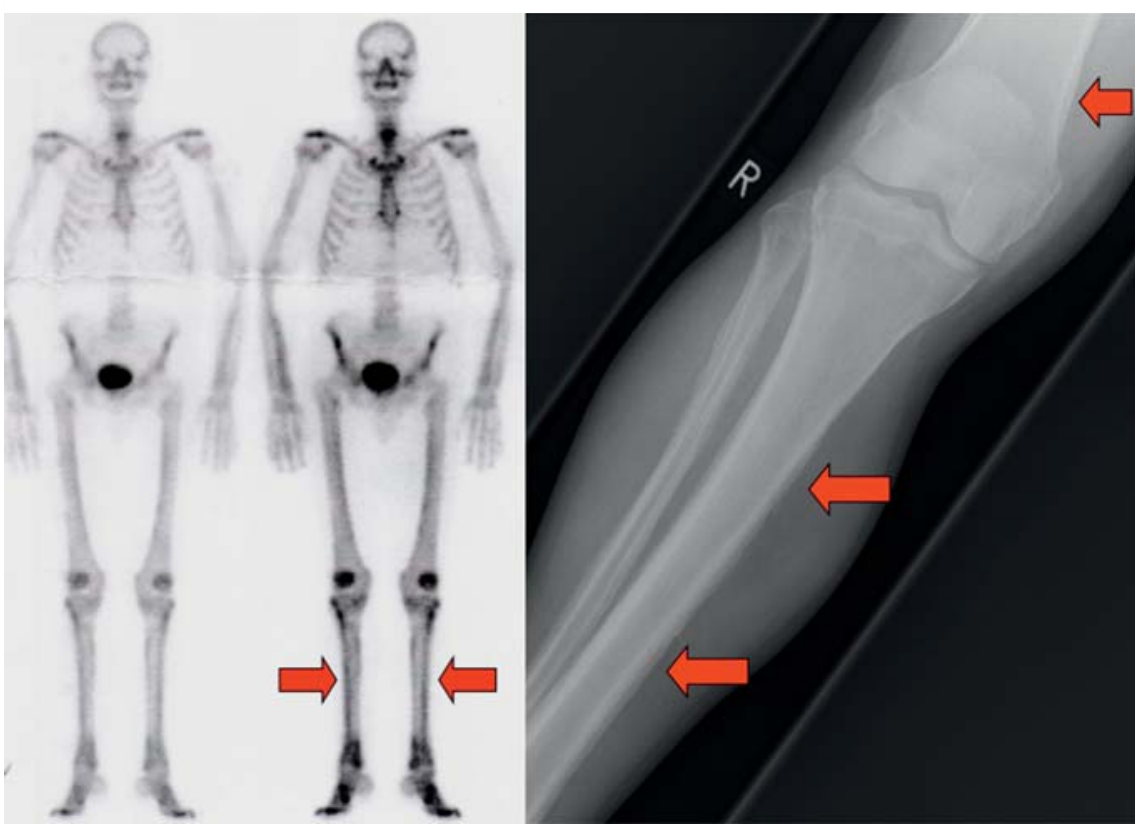

Abb. 3 Skelettszintigrafiebefund und Röntgenbefund des Patienten im Fall Nr. 1, vor Beginn der Therapie. Die Pfeile zeigen die typischen PeriostAnreicherungen in der Skelettszintigrafie und die typische Periostverdickung im Röntgenbild. 

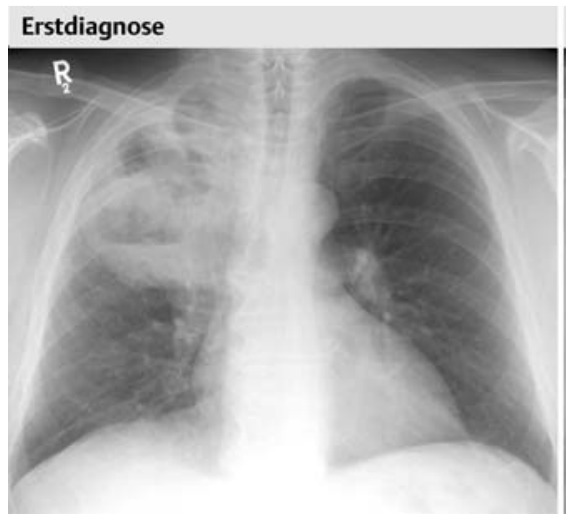

nach Radiochemotherapie

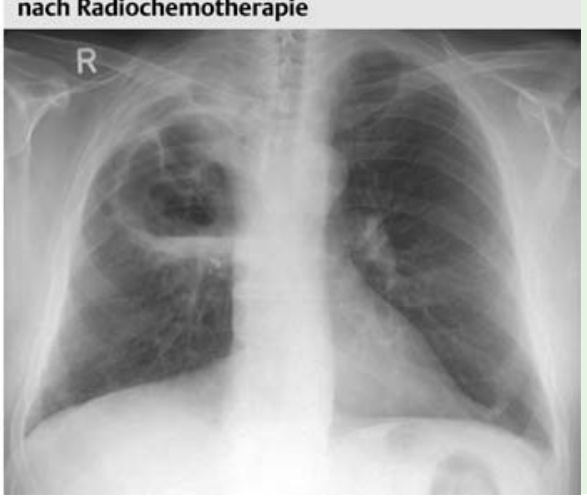

Abb.4 Röntgen-Thorax des Patienten im Fall Nr. 1, vor Beginn der Therapie und nach 6-monatiger Therapie (Radiochemotherapie und nachfolgende ambulante Chemotherapie).
Seit 7 Monaten vor Diagnosestellung wurde das Auftreten von Trommelschlegelfingern bemerkt, kurz darauf traten Knochenund Gelenkbeschwerden, insbesondere Schultergelenksbeschwerden, auf. Diese wurden mit NSAR behandelt (mit mäßigem Erfolg) und nicht weiter abgeklärt. Seit 4 Monaten vor Diagnosestellung wurde eine Paraparese des rechten Beins und Arms bemerkt, begleitet von einer Fallneigung. Eine neurologische Fachabteilung stellte aufgrund eines positiven Serum-IgM-Titers gegen Borrelia burgdorferi (bei sonst fehlenden Hinweisen auf eine Borreliose) die Diagnose Neuroborreliose, ein MRT-Kopf wurde nicht durchgeführt. Eine 14-tägige Ceftriaxon-Therapie blieb ohne klinischen Erfolg. Eine nachfolgende Abklärung bei einem niedergelassenen Orthopäden erbrachte keine Auffälligkeiten. Bei zunehmender Parese des rechten Arms und Beins erfolgte eine Vorstellung bei einem niedergelassenen Neurologen, der ein MRT des Gehirns veranlasste. Dieses zeigte eine multiple Hirnmetastasierung, der größte Herd zeigte sich links subkortikal, mit ausgeprägter Ödembildung. Kurz darauf wurde die Patientin aufgrund eines fokalen Krampfanfalls in eine neurochirurgische Klinik aufgenommen. Es wurde ein CT des Thorax veranlasst, welches eine ausgedehnte pulmonale Raumforderung rechts mit Einwachsen in das Mediastinum erbrachte. Im CT des Abdomens fand sich kein Anhalt für einen abdominellen Focus oder Metastasierung. Es wurde entschieden, die Histologie mittels Kraniotomie aus einer zerebralen Metastase zu gewinnen, diese erbrachte ein Adenokarzinom (G3), am ehesten pulmonalen Ursprungs.

Daraufhin wurde die Patientin in unsere Klinik verlegt. Die Patientin verneinte respiratorische Beschwerden bzw. Dyspnoe, blutgasanalytisch fand sich keine respiratorische Insuffizienz. Skelettszintigrafisch fand sich kein Hinweis auf ossäre Metastasen, jedoch ein Anreichungsmuster vereinbar mit einer hypertrophen Osteoarthropathie. Die Histologie der bronchoskopisch entnommenen Zangenbiopsien bestätigte ein Adenokarzinom (G3), die EGFR-Mutationsanalyse erbrachte keine Mutation im EGFRGen. Es wurde ein NSCLC mit der Tumorformel T4N3M1b diagnostiziert. Nach simultaner Radio-Chemotherapie wurden insgesamt 6 Zyklen Chemotherapie mit Carboplatin und Pemetrexed appliziert. Im nachfolgenden Re-Staging zeigte sich ein TumorRegress. Nach insgesamt 4-monatiger Therapiepause kam es jedoch zu einem lokalen Tumorprogress, zudem traten Weichteilmetastasen auf, sodass nun eine Second-Line-Chemotherapie begonnen werden soll.

\section{Fall 3}

\section{$\nabla$}

Es handelt sich um einen 46-jährigen Mann, der 10 Jahre als Schiffbauer in einer Rostocker Werft gearbeitet hatte und eine Asbestexposition in diesem Zusammenhang verneinte. Er verlor seinen Arbeitsplatz im Rahmen der deutschen Wiedervereinigung und arbeitete seit 16 Jahren in einem holzverarbeitenden Kleinbetrieb. Er hatte bis zuletzt 35 PY geraucht. Eine Alkoholabhängigkeit bestand bis vor 2 Jahren. Vorerkrankungen waren ein Hypertonus, eine chronische Gastritis, eine Laktoseintoleranz und diverse Nahrungsmittelallergien, die Dauermedikation bestand aus Bisoprolol, Eprosartan und Lansoprazol. Seit 3 Monaten vor Diagnosestellung bemerkte der Patient einen Leistungsknick, Gewichtsverlust, Knochen- und Gelenkbeschwerden (insbesondere Schmerzen in den Kniegelenken und Ellenbogen-Gelenken) und die Ausbildung von Trommelschlegelfingern. Bezüglich der Gelenkbeschwerden glaubte der Patient, dass diese vom vielen Schneeschieben im Winter herrührten, sie nahmen aber nach Ende der Schneeperiode weiter zu. Seit 4 Wochen vor Diagnosestellung trat Nachtschweiß auf. Seitens des Hausarztes war der Patient zunächst nur aufgefordert worden, „mehr zu essen“. Aufgrund progredienter B-Symptomatik wurde eine elektive Gastroskopie, aufgrund der Knieschmerzen ein Vorstellungstermin bei einem Orthopäden vereinbart.

Der Patient stellte sich in unserer Klinik als Notfall aufgrund zunehmenden Schwindels (mit Fallneigung) und Oberbauchschmerzen vor. Der Patient verneinte respiratorische Beschwerden bzw. Dyspnoe. Die Gastroskopie zeigte eine Pangastritis, Duodenitis und Refluxösophagitis. Aufgrund der Symptomkonstellation Trommelschlegelfinger und Gelenkschmerzen wurden ein Röntgen-Thorax und ein CT-Thorax durchgeführt, welches eine große Raumforderung im rechten Unterlappen, kontralaterale Metastasen und mediastinale Lymphknotenschwellungen zeigte ( $\bullet$ Abb.5). Histologisch wurde ein großzelliges Karzinom der Lunge (G3) gesichert, die EGFR-Mutationsanalyse erbrachte keine Mutation im EGFR-Gen. Zusätzlich wurden große Nebennieren-Metastasen bds. und eine solitäre Hirnmetastase rechts occipital nachgewiesen. Skelettszintigrafisch fand sich kein Hinweis auf ossäre Metastasen, jedoch ein Anreichungsmuster vereinbar mit einer hypertrophen Osteoarthropathie. Es wurde ein NSCLC mit der Tumorformel T4N3M1b diagnostiziert. Eine Radiatio des Kopfes lehnte der Patient ab, sodass eine alleinige Chemotherapie mit Cisplatin und Gemcitabine begonnen wurde. Aufgrund der Nebenwirkungen der Therapie brach der Patient nach 3 Zyklen die Chemotherapie ab und verstarb einige Wochen später in einem Hospiz. 


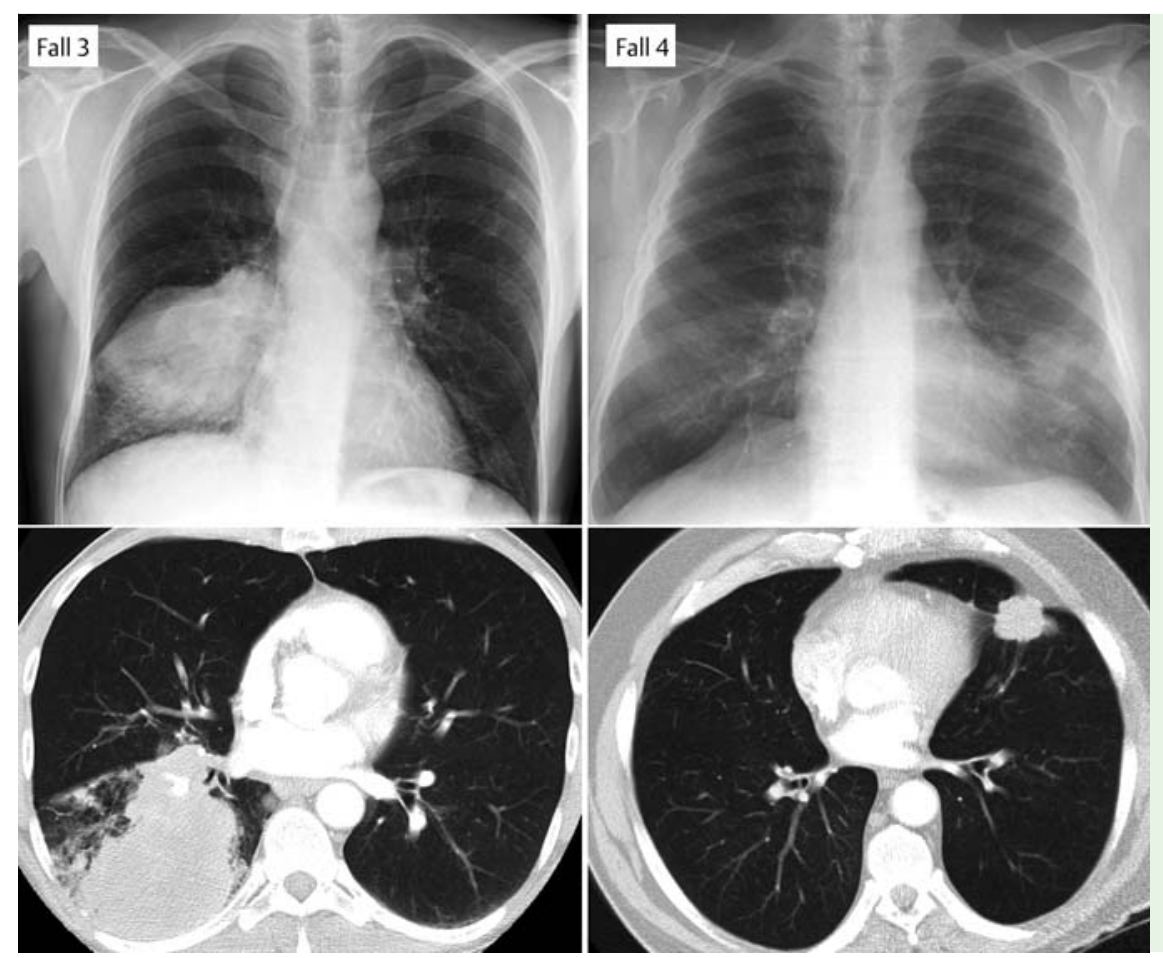

Abb. 5 Röntgen-Thorax und CT-Thorax der Patienten der Fälle Nr. 3 und 4, vor Beginn der Therapie.

\section{Fall 4}

$\nabla$

Es handelt sich um einen 70-jährigen Mann, der über 20 Jahre Bauer in einer Landwirtschaftlichen Produktionsgenossenschaft (LPG) war und seit 1975 hauptsächlich Bürotätigkeiten nachging (als Mitarbeiter der sogenannten Agrarindustriellen Vereinigung in der DDR). Nach der Wiedervereinigung Deutschlands arbeitete er nach einer Umschulung als Fahrlehrer. Er hatte bis vor 3 Jahren insgesamt 55 PY geraucht. Ein Zittern der Hände wurde seit 10 Jahren bemerkt, es wurde vor 3 Jahren der Verdacht auf Morbus Parkinson geäußert, eine diesbezügliche Therapie erfolgte jedoch nicht. Seit 15 Jahren nutzt der Patient regelmäßig eine CPAP-Therapie zur Behandlung seines obstruktiven Schlafapnoesyndroms. Eine COPD im Stadium II nach GOLD wurde seit einigen Jahren mit Formoterol und Tiotropium behandelt. Drei Monate vor Diagnosestellung bemerkte der Patient eine Schwellung und Rötung der Fingerendglieder, einen Monat später auch der Großzehen und der 2. Zehen ( $\bullet$ Abb. 6 und $\bullet$ Abb.7). Aufgrund der Fingerveränderungen konnte er Zettel nicht mehr vom Boden aufheben, auch das Blättern in Zeitungen fiel ihm schwer. Zudem war das Druckgefühl in den Fingerkuppen lästig. Eine seit Jahrzehnten aufgetretene (und in der Familie des Patienten wiederholt auftretende) Längsspaltung der Fingernägel (Onychodystrophie) verschwand plötzlich, die Nägel waren jetzt zwar abgerundet, aber intakt. Knochenschmerzen oder Gelenkschmerzen wurden verneint. Seit 2 Monaten vor Diagnosestellung kam es zu einem zunehmenden trockenen Husten (jedoch nicht zu einer zunehmenden Luftnot) und zu einer submandibulären Lymphknotenschwellung links. Aufgrund der lästigen Fingerveränderungen und des Hustens suchte der Patient seinen Hausarzt auf, der „nichts zu den Fingern sagen konnte“ und bezüglich des Hustens dem Patienten ein Antitussivum verschrieb.

Aufgrund des weiter zunehmenden Hustens ließ sich der Patient Überweisungen zu diversen Fachärzten ausstellen, bekam dort aber erst Termine in 3-6 Monaten. Daher stellte sich der Patient in Eigeninitiative in der Notaufnahme unserer Klinik vor. Im

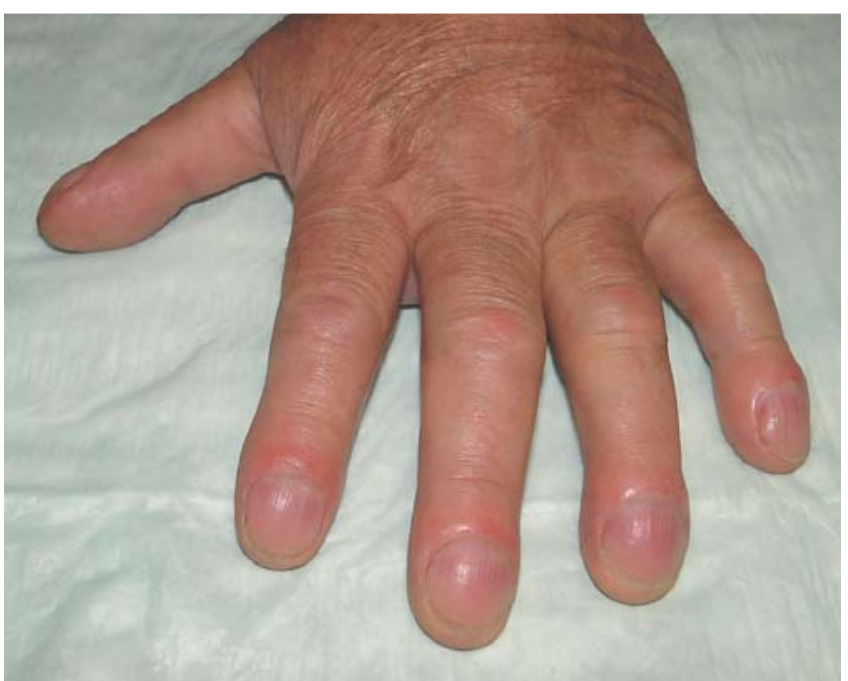

Abb. 6 Hand des Patienten im Fall Nr. 4.

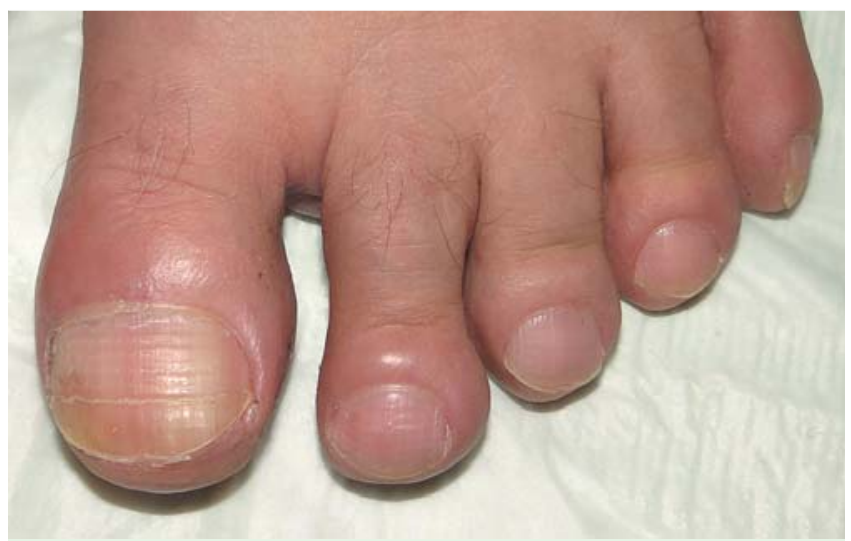

Abb.7 Fuß des Patienten im Fall Nr. 4. 
Röntgen-Thorax und CT-Thorax fand sich eine Raumforderung links ( Abb.5), histologisch ein Adenokarzinom G2, ohne Nachweis einer Mutation im EGFR-Gen. Es wurden pulmonale Metastasen und eine submandibuläre Lymphknotenmetastase links nachgewiesen. Es fand sich kein Anhalt für abdominelle, ossäre oder zerebrale Metastasen. Es wurde ein NSCLC mit der Tumorformel T4N2M1b festgestellt. Skelettszintigrafisch fand sich das typische Anreichungsmuster einer hypertrophen Osteoarthropathie, obgleich der Patient weiterhin keine Knochen- und Gelenkschmerzen angab. Nach 6 Zyklen Chemotherapie mit Carboplatin/Pemetrexed zeigte sich im Re-Staging eine leichte Größenregredienz des Tumors und der Metastasen. Die Finger- und Zehenveränderungen persistierten. Es wurde eine ALK-Mutationsdiagnostik veranlasst und auf Wunsch des Patienten eine zweimonatige Therapiepause vereinbart.

\section{Diskussion}

$\nabla$

Die 4 hier vorgestellten Fälle haben folgendes gemeinsam:

- Die Ausbildung der Trommelschlegelfinger und der Gelenkund Knochenschmerzen gingen der Diagnose der Tumorerkrankung um mehrere Monate voraus.

- In der Phase der Ausbildung der paraneoplastischen Symptome waren die Patienten respiratorisch beschwerdefrei und nicht hypoxisch.

- Das Neu-Auftreten von Trommelschlegelfingern wurde von den primär behandelnden Ärzten nicht als möglicher Fingerzeig auf ein NSCLC erkannt: eine frühe Bildgebung des Thorax blieb aus.

Es gab jedoch auch Unterschiede zwischen den hier vorgestellten Fällen:

- Bei einem Patient (70 Jahre) war das Alter bei Erstdiagnose im typischen Bereich der Bronchialkarzinome in Deutschland [4], die anderen drei Patienten waren vergleichsweise jung (44, 46 und 58 Jahre alt).

- Bei einem Patienten traten keine Gelenk- oder Knochenschmerzen auf, obgleich der typische Befund einer hypertrophen Osteoarthropathie skelettszintigrafisch nachweisbar war (inwieweit diese Diskrepanz an dem vermuteten M. Parkinson liegt, ist unklar).

- In 3 Fällen lagen Adenokarzinome vor, bei einem Fall wurde ein großzelliges Karzinom diagnostiziert.

- Die pulmonalen Raumforderungen waren unterschiedlicher Größe und Form, sodass keine charakteristische pulmonale Tumormanifestation den Fällen gemeinsam war.

Die hypertrophe pulmonale Osteoarthropathie, welche (in ihrer kompletten Form) klinisch durch das gleichzeitige Auftreten von Knochen- und Gelenkschmerzen sowie von Trommelschlegelfingern gekennzeichnet ist, wurde erstmals 1889 durch den österreichischen Internisten Eugen von Bamberger [5] und 1890 durch den französischen Neurologen Pierre Marie [6] beschrieben und wird daher auch als Pierre-Marie-Bamberger-Syndrom oder Marie-Bamberger-Syndrom bezeichnet (die letztere Bezeichnung ist logischer, da sie nur die Nachnamen der beiden Erstbeschreiber enthält). Diese Erstbeschreibungen handelten von Patienten mit Bronchiektasie [5] und von Patienten mit Lungentuberkulose [6], somit also von Patienten mit benignen Lungenerkrankungen. Die hypertrophe pulmonale Osteoarthropathie wurde in der Tat in der ersten Jahrzehnten nach Erstbeschreibung vor allem bei benignen (meist raumfordernden) Lungenerkrankungen (Lungentuberkulose, Lungenabszess, Bronchiektasie etc.) beschrieben
[7]. Später wurden zunehmend diverse im Thorax auftretende Tumore (nichtkleinzellige Bronchialkarzinome, Lymphome, Thymome, Fibrome, Metastasen etc.) als weitere Ursachen des Marie-Bamberger-Syndroms beschrieben $[8,9]$. Aufgrund der sinkenden Zahl an raumfordernden infektiologischen Lungenerkrankungen (wie der Lungentuberkulose) und der steigenden Zahl an Bronchialkarzinomen ist das nichtkleinzellige Bronchialkarzinom (NSCLC) in den letzten Jahrzehnten zur führenden Ursache (und somit auch zur führenden Differentialdiagnose) des Marie-Bamberger-Syndroms in den westlichen Industrienationen aufgestiegen [1,2]. Wiewohl die Ausbildung einer Osteoarthropathie und von Trommelschlegelfingern bei chronischen Darm-Erkrankungen wie der Colitis ulcerosa oder dem Morbus Crohn $[10,11]$ und bei chronischen Lebererkrankungen [12 - 14] beschrieben wurde, ist das gemeinsame Auftreten beider Symptome (als komplettes Syndrom) bei diesen Patienten eher selten. Somit gilt das NSCLC heutzutage als wichtigste Differentialdiagnose des Marie-Bamberger-Syndroms. Zu trennen ist das MarieBamberger-Syndrom von pulmonalen und kardialen Erkrankungen, bei denen es im Rahmen einer chronischen Hypoxie zu Trommelschlegelfingern kommt. Typisch für das Marie-Bamberger-Syndrom als früher NSCLC-Hinweis ist das Fehlen einer signifikanten Hypoxie, die Finger sind nicht zyanotisch.

Über das Auftreten des Marie-Bamberger-Syndroms beim NSCLC gibt es viele Fallberichte, man geht von einer Inzidenz von 1-5\% aller Patienten mit NSCLC aus $[2,9,15,16]$. Bezüglich eines für ein Marie-Bamberger-Syndrom möglicherweise prädisponierenden NSCLC-Erkrankungs-Alters finden sich in der Literatur keine dezidierten Aussagen, es fällt jedoch in den Fallberichten auf, dass die meisten Patienten relativ jung sind. So sind die 7 berichteten paraneoplastischen Fälle von Vogl et al. zum Beispiel zwischen 44 und 58 Jahre alt. Diese Altersverteilung stimmt auffällig mit 3 der 4 hier beschriebenen Fälle überein (auch zwischen 44-58 Jahre alt). In einer der größten Fallserien von Semple et al. mit 24 NSCLC-Patienten lag das durchschnittliche Alter bei 50 Jahren, nur 2 von 24 Patienten waren über 60 Jahre alt [17]. Somit scheint es einen gewissen Schwerpunkt dieses paraneoplastischen Syndroms bei jüngeren NSCLC-Patienten zu geben. Die Gründe hierfür sind unklar. Bezüglich der Geschlechtsverteilung wurden in der Literatur vor allem Männer mit Marie-BambergerSyndrom bei NSCLC beschrieben. Dies könnte jedoch an der (bisherigen) generellen Geschlechtsverteilung beim NSCLC liegen und sich folglich in den nächsten Jahren (mit zunehmender NSCLC-Inzidenz bei Frauen) ändern [7].

Bezüglich der hypertrophen Osteoarthropathie kann die Diagnose durch Röntgenaufnahmen der langen Röhrenknochen, die eine charakteristische Periostverdickung zeigen, erhärtet werden ( Abb.3). Die Skelettszintigrafie gilt jedoch bis heute als beste und etablierteste Nachweismethode ( $\bullet$ Abb.3). Die spezifischen skelettszintigrafischen Veränderungen beinhalten vor allem eine charakteristische periostale Anreicherung im Bereich der langen Röhrenknochen [18]. Jüngste Berichte zeigen, dass auch mittels PET-CT charakteristische Skelett-Veränderungen im Sinne eines Marie-Bamberger-Syndroms nachgewiesen werden können (FDG-Anreicherung im Bereich des Periosts der langen Röhrenknochen) [19,20].

Die Pathogenese des Marie-Bamberger-Syndroms ist bis heute nicht sicher. Wiewohl unbestritten ist, dass ein raumfordernder Prozess im Thorax der Erkrankung zugrunde liegt, ist der pathogenetische Zusammenhang zwischen dem Lungenprozeß einerseits und den Trommelschlegelfingern bzw. der Periostverdi- 
ckung andererseits bis heute umstritten. Es existieren drei führende Hypothesen:

1. Neuronale Hypothese: Es wurde in verschiedenen Einzel-Beschreibungen gezeigt, dass die Durchtrennung des Nervus vagus auf der betroffenen Seite zu einem raschen Rückgang (innerhalb von Stunden) der Symptome eines Marie-BambergerSyndroms führen kann $[9,21,22]$. Es wird ein zentraler neuronaler Reflex vermutet, der über den N. vagus (der neben parasympathischen Fasern fast alle sensorischen Fasern der Lunge enthält) zur sympathischen Innervation peripherer Blutgefäße führt und über eine Veränderung der Gefäßweite und Gefäßpermeabilität zur Periostverdickung und zur Ausbildung von Trommelschlegelfingern führt. Bewiesen wurde diese Hypothese bislang nicht. Die Hypothese wird allerdings durch den Umstand unterstützt, dass fast alle bislang beschriebenen Auslöser des Marie-Bamberger-Syndroms im Innervationsgebiet des N. vagus liegen.

2. Humorale Hypothese: Diese Hypothese besagt, dass durch die Lungenprozesse Wachstumsfaktoren in die Blutbahn sezerniert werden, die zu den Periost- und Weichteilveränderungen des Marie-Bamberger-Syndroms führen [9,23]. Die humorale Hypothese ist die am wenigsten plausible Hypothese, da es zwei Gegenargumente gibt. Einerseits ist es aufgrund der Diversität an möglichen auslösenden benignen und malignen Lungenprozessen des Marie-Bamberger-Syndroms schwer vorstellbar, dass ein charakteristischer Botenstoff (oder eine charakteristische Gruppe an Botenstoffen) von allen Prozessen sezerniert wird. Desweiteren bleibt bei dieser Hypothese unerklärt, warum die Veränderungen nur an bestimmten Stellen des Skelettsystems (und nicht etwa am gesamten Skelett) auftreten.

3. Megakaryozytäre Hypothese: Diese Hypothese geht von der (bislang nicht bewiesenen) Grundannahme aus, dass Megakaryozyten nicht im Knochenmark, sondern in der Lungenstrombahn zu Thrombozyten zerfallen. Laut dieser Hypothese werden die Megakaryozyten aus dem Knochenmark in die Blutbahn ausgeschwemmt, bleiben in den Lungenkapillaren stecken und zerfallen dort in Thrombozyten. Im Rahmen eines arteriovenösen Shunts, der durch die raumfordernden Lungenprozesse ausgelöst wird, bleibt der MegakaryozytenZerfall aus, und es gelangen Megakaryozyten in die Kapillaren der Peripherie. Dort könnten sie durch Freisetzung von Wachstumsfaktoren und anderen Mediatoren ein MarieBamberger-Syndrom auslösen [24,25]. Bewiesen wurde auch diese Hypothese bislang nicht.

Die Literatur stimmt darüber überein, dass die Symptome des Marie-Bamberger-Syndroms bei erfolgreicher Therapie des NSCLC rückläufig sind $[2,8,25]$. In dem ersten hier beschriebenen Casus waren die Symptome nicht rückläufig, trotz guter zwischenzeitlicher Tumorkontrolle nach Radiochemotherapie. Es persistierte jedoch eine große Kaverne im Thorax. Folgt man der neuronalen oder megakaryozytären Hypothese, dann ist nicht das Vorhandensein eines NSCLC, sondern die pulmonale Strukturveränderung entscheidend für das Marie-Bamberger-Syndrom. Somit würden diese beiden Hypothesen erklären, warum im Fall 1 das paraneoplastische Syndrom persistierte. Neben der kausalen Therapie des Marie-Bamberger-Syndroms (Bekämpfung der pulmonalen Raumorderung) und der oben beschriebenen Vagotomie $[9,21,22]$ wurden in den letzten Jahren verschiedene medikamentöse Therapiestrategien vorgeschlagen [26]. Dazu gehören diverse Nichtsteroidale Antirheumatika (NSAR) $[27,28]$, Bisposphonate $[29,30]$, das Somatostatin-Analogon Oct- reotid [31,32] und der VEGF-Inhibitor Bevacizumab [23,33]. Eine allgemein akzeptierte Therapieempfehlung gibt es bislang aber nicht.

Auffällig war in allen hier beschriebenen Fällen, dass die Ausbildung der Trommelschlegelfinger der NSCLC-Diagnosestellung vorausging. Sie traten in einer Phase auf, in der die Patienten noch respiratorisch beschwerdefrei waren (bei 2 von 4 Patienten zeichnete sich jedoch bereits eine B-Symptomatik ab). Keiner der primär behandelnden Ärzte erkannte die typische Symptomkonstellation, es wurde daher keine Bildgebung des Thorax initiiert. Somit wurde in allen Fällen die Chance einer Diagnosestellung in einem früheren NSCLC-Tumorstadium vertan. Dies ist auch in der Literatur schon häufig beschrieben worden. Das Anliegen der Fallberichte und Übersichtsarbeiten in der Literatur zu diesem paraneoplastischen Syndrom, wie auch der vorliegenden Arbeit, ist es daher immer wieder, auf diese „paraneoplastische Chance" aufmerksam zu machen. Das Neu-Auftreten von Trommelschlegelfingern (und begleitend eventuell sogar Knochen- und Gelenkbeschwerden) muss eine umgehende Thoraxbildgebung auslösen: Dies kann nicht oft genug betont werden.

\section{Interessenkonflikt}

$\nabla$

Die Autoren geben an, dass kein Interessenkonflikt besteht.

\section{Literatur}

1 Ray RS, Fisher HP Jr . Hypertrophic osteoarthropathy in pulmonary malignancies. Ann Intern Med 1953; 38: 239-246

2 Ito T, Goto K, Yoh K et al. Hypertrophic pulmonary osteoarthropathy as a paraneoplastic manifestation of lung cancer. J Thorac Oncol 2010; 5: 976-980

3 Pankow W, Neumann K, von Wichert P. [Bronchial carcinoma associated with pulmonary osteoarthropathy (Marie-Bamberger)]. Pneumologie 1990; 44: $1306-1311$

4 Goeckenjan G, Sitter H, Thomas M et al. Prevention, diagnosis, therapy, and follow-up of lung cancer: interdisciplinary guideline of the German Respiratory Society and the German Cancer Society. Pneumologie 2011; 65: 39-59

5 von Bamberger E. Protokoll der K. K. Gesellschaft der Aerzte in Wien. Sitzung vom 8. März 1889. Wien Klin Wochenschr 1889; 2: 226

6 Marie P. De l'osteoarthropathie hypertrophiante pneumique. Rev de méd 1990; 10: 1 -1

7 Benedek TG. Paraneoplastic digital clubbing and hypertrophic osteoarthropathy. Clin Dermatol 1993; 11: 53-59

$8 \mathrm{Vogl} \mathrm{A}$, Blumenfeld S, Gutner LB. Diagnostic significance of pulmonary hypertrophic osteoarthropathy. Am J Med 1955; 18: 51 -65

9 Goldstraw P, Walbaum PR. Hypertrophic pulmonary osteoarthropathy and its occurrence with pulmonary metastases from renal carcinoma. Thorax 1976; 31: 205-211

10 Herneth AM, Breitenseher MJ, Funovics $M$ et al. Quiz case 12. MarieBamberger syndrome (MBS) (hypertrophic osteoarthropathy (HOA) secondary to ulcerative colitis (UC). Eur J Radiol 1999; 32: 124-128

11 Kitis G, Thompson H, Allan RN. Finger clubbing in inflammatory bowel disease: its prevalence and pathogenesis. Br Med J 1979; 2: 825-828

12 Huaux JP, Geubel A, Maldague B et al. Hypertrophic osteoarthropathy related to end stage cholestatic cirrhosis: reversal after liver transplantation. Ann Rheum Dis 1987; 46: 342 - 345

13 Taillandier J, Alemanni M, Samuel D et al. Hepatic hypertrophic osteoarthropathy: the value of liver transplantation. Clin Exp Rheumatol 1998; 16: 80-81

14 Katsicas $M$, Ciocca M, Rosanova $M$ et al. Hypertrophic osteoarthropathy in two children with cholestatic hepatic disease. Acta Paediatr 2005; 94: $1152-1155$

15 Izumi M, Takayama K, Yabuuchi $H$ et al. Incidence of hypertrophic pulmonary osteoarthropathy associated with primary lung cancer. Respirology 2010; 15: 809-812

16 Segal AM, Mackenzie AH. Hypertrophic osteoarthropathy: a 10-year retrospective analysis. Semin Arthritis Rheum 1982; 12: 220-232 
17 Semple T, McCluskie RA. Generalized hypertrophic osteoarthropathy in association with bronchial carcinoma; a review, based on 24 cases. $\mathrm{Br}$ Med J 1955; 1: 754-759

18 Koischwitz D, Dewes W, Bahre $M$ et al. [Correlation of scintigraphic and $\mathrm{x}$-ray findings in Marie-Bamberger secondary hypertrophic osteoarthropathy]. Rofo 1986; 144: 681-688

19 Strobel K, Schaefer NG, Husarik DB et al. Pulmonary hypertrophic osteoarthropathy in a patient with nonsmall cell lung cancer: Diagnosis with FDG PET/CT. Clin Nucl Med 2006; 31: 624-626

20 Makis W, Abikhzer G, Rush C. Hypertrophic pulmonary osteoarthropathy diagnosed by FDG PET-CT in a patient with lung adenocarcinoma. Clin Nucl Med 2009; 34: 625-627

21 Treasure $T$. Hypertrophic pulmonary osteoarthropathy and the vagus nerve: an historical note. J R Soc Med 2006; 99: 388-390

22 Flavell G. Reversal of pulmonary hypertrophic osteoarthropathy by vagotomy. Lancet 1956; 270: 260-262

23 Atkinson S, Fox SB. Vascular endothelial growth factor (VEGF)-A and platelet-derived growth factor (PDGF) play a central role in the pathogenesis of digital clubbing. J Pathol 2004; 203: 721 - 728

24 Dickinson CJ. The aetiology of clubbing and hypertrophic osteoarthropathy. Eur J Clin Invest 1993; 23: 330-338

25 Fietz T, Schneider P, Knauf $W U$ et al. [Clubbed fingers and arthralgia as a reversible paraneoplastic syndrome (Pierre-Marie-Bamberger syndrome) in non-small-cell bronchial carcinoma]. Dtsch Med Wochenschr 1998; 123: $1507-1511$
26 Nguyen S, Hojjati M. Review of current therapies for secondary hypertrophic pulmonary osteoarthropathy. Clin Rheumatol 2011; 30: 7-13

27 Leung FW, Williams AJ, Fan P. Indomethacin therapy for hypertrophic pulmonary osteoarthropathy in patients with bronchogenic carcinoma. West J Med 1985; 142: 345-347

28 Blackwell N, Bangham L, Hughes $M$ et al. Treatment of resistant pain in hypertrophic pulmonary arthropathy with ketorolac. Thorax 1993; 48: 401

29 Speden $D$, Nicklason $F$, Francis $H$ et al. The use of pamidronate in hypertrophic pulmonary osteoarthropathy (HPOA). Aust N Z J Med 1997; 27 : 307-310

30 King MM, Nelson DA. Hypertrophic osteoarthropathy effectively treated with zoledronic acid. Clin Lung Cancer 2008; 9: 179-182

31 Johnson SA, Spiller PA, Faull CM. Treatment of resistant pain in hypertrophic pulmonary osteoarthropathy with subcutaneous octreotide. Thorax 1997; 52: $298-299$

32 Angel-Moreno Maroto A, Martinez-Quintana E et al. Painful hypertrophic osteoarthropathy successfully treated with octreotide. The pathogenetic role of vascular endothelial growth factor (VEGF). Rheumatology (Oxford) 2005; 44: 1326-1327

33 Olan F, Portela $M$, Navarro $C$ et al. Circulating vascular endothelial growth factor concentrations in a case of pulmonary hypertrophic osteoarthropathy. Correlation with disease activity. J Rheumatol 2004; 31: $614-616$ 